F043, version 2.02, 30 August 2001

\title{
The effect of the cell size in Langevin micromagnetic simulations
}

\author{
V. Tsiantos ${ }^{\mathrm{a}}$, W. Scholz ${ }^{\mathrm{a}}$, D. Suess ${ }^{\mathrm{a}}$, T. Schrefl ${ }^{\mathrm{a}}$, J. Fidler $^{\mathrm{a}}$ \\ ${ }^{a}$ Institute of Applied and Technical Physics, Vienna University of Technology, \\ Wiedner Hauptstr. 8-10, A-1040 Vienna, Austria.
}

\begin{abstract}
Langevin micromagnetics treats finite temperature effects by adding a thermal fluctuation field, $H_{\mathrm{th}}$, to the effective field. If combined with the finite element method or the finite difference method, the spatial correlation length of the random field is usually taken to be equal to the cell size of the computational grid. The influence of the cell size has been studied for two different systems: a system close to equilibrium and a system which exhibits thermal activated switching. The results suggest that the calculated properties are independent of the cell size if the cell size is smaller than the thermal exchange length $\sqrt{A /\left(J_{s} H_{\text {th }}\right)}$. The term $A$ is the exchange constant and $J_{\mathrm{s}}$ is the spontaneous magnetic polarization. Below this critical length the magnetization is uniform.
\end{abstract}

Keywords: numerical micromagnetics, Langevin equation, thermal activation.

Corresponding author: V. D. Tsiantos, Vienna University of Technology, Institute of Applied and Technical Physics, Wiedner Hauptstrasse 8-10/137, A-1040, Vienna, Austria. FAX:

0043-1-58801 13798. Email: v.tsiantos@computer.org 


\section{Introduction}

Thermally activated magnetization reversal is an important issue in high density recording. With decreasing bit size thermally induced switching may influence the write process as well as the long term stability of written bits. The theoretical treatment of thermal process on the small time scale usually starts from the Langevin equation [1-3]. Although the details of the implementations differ all authors [1-3] apply the fluctuation dissipation theorem in order to derive the strength of a random thermal field which is then added to the effective field in the Gilbert equation of motion. If system under investigation consists of small individual particles [2] each particle is assumed to stay uniformly magnetized during magnetization reversal. The thermal fluctuation field acts on the entire particle.

In order to treat magnetization processes in larger particles, the particles are generally subdivided into smaller computational cells. The spatial correlation length of the thermal fluctuation is chosen to coincide with the size of the computational cell. Though this might be a convenient assumption it has two major implications. First, the spatial correlation length of the thermal noise changes with the mesh size. As the cell is reduced to obtain higher spatial resolution, for example near sharp edges or corners, the thermal noise changes as well. Second, Langevin dynamics derived from a corresponding Fokker-Planck equation assumes white noise [4], with the thermal fluctuation being uncorrelated in space and time. The finite cell size which equals the correlation length may lead to a crude approximation of white noise.

This paper starts from two numerical examples investigating the influence of cell size on the numerical results obtained from the solution of the Langevin equation. Thermal equilibrium properties such as the average magnetization at a given temperature and the relaxation time 
over an energy barrier are calculated for a small cube of Co. The results are found to be independent of the mesh size if the mesh size is small than a characteristic length. This thermal exchange length gives the characteristic length on which fluctuations of the magnetization, due to a single thermal event, decay in space.

\section{Langevin micromagnetics}

The theoretical treatment of thermally activated magnetization reversal for particles with an extension greater than the exchange length requires to solve the Langevin equation numerically. The Langevin equation follows from the Gilbert equation of motion by adding a random thermal fluctuation field to the effective magnetic field:

$$
\frac{\partial \mathbf{J}}{\partial t}=-\gamma \mid \mathbf{J} \times\left(\mathbf{H}_{e f f}+\mathbf{H}_{t h}\right)+\frac{\alpha}{J_{s}} \mathbf{J} \times \frac{\partial \mathbf{J}}{\partial t}
$$

The first term on the right hand side of equation (1) accounts for the gyromagnetic precession of the magnetic polarization $\mathbf{J}$, the second term arises from viscous damping. The term $\gamma$ is the gyromagnetic ratio, and $\alpha$ is the Gilbert damping constant. The thermal field is assumed to be a Gaussian random process with the following statistical properties:

$$
\left\langle\boldsymbol{H}_{t h, i}^{k}, \boldsymbol{H}_{t h, j}^{l}\right\rangle=2 D \delta_{i j} \delta_{k l} \delta\left(t-t^{\prime}\right)
$$

The average of the thermal field taken over different realizations vanishes in each direction $i$ in space. The thermal field is uncorrelated in time and uncorrelated at different node points 
$(k, l)$ of the finite element mesh. The strength of the thermal fluctuations follows from the fluctuation-dissipation theorem:

$$
D=\frac{\alpha k_{B} T}{\gamma J_{s} l^{3}}
$$

where $l$ is the cell size, and $k_{\mathrm{B}}$ is the Boltzman constant.

\section{Results and discussion}

Two characteristic length scales are important in ferromagnetic materials: The exchange length,

$$
l_{\mathrm{ex}}=\sqrt{\frac{2 \mu_{0} A}{J_{s}^{2}}},
$$

and the Bloch parameter

$$
\delta_{0}=\sqrt{\frac{A}{K}}
$$

Here $A$ is the exchange constant, $J_{\mathrm{s}}$ is the spontaneous magnetic polarization, and $K$ is the magnetocrystalline anisotropy constant. These length scales determine the width of magnetic inhomogeneities. Generally, the magnetization will change over a length of $\pi l_{\text {ex }}$ or $\pi \delta_{0}$ in a Neel wall or Bloch wall, respectively. In order to resolve this transition of the magnetization, the computational cells have to be smaller than the minimum of $l_{\mathrm{ex}}$ and $\delta_{0}$. Rave and coworkers [5] clearly demonstrated the convergence of the numerical solution if the cell size becomes smaller than the exchange length. At zero temperature the interplay between exchange interactions and the demagnetizing field or the interplay between exchange and magnetocrystalline anisotropy causes the magnetization to change its direction over a length 
given by $\pi l_{\mathrm{ex}}$ or $\pi \delta_{0}$. At finite temperature thermal noise may cause the magnetization to rotate out of its preferred orientation. This will cause a change of the magnetization not only on the lattice site where the magnetization was randomly kicked by the thermal field but also in its surroundings. The fluctuation of the magnetization due to the thermal event extends over a characteristic length which is now governed by the interplay between the exchange interactions and the strength of the thermal field. We can define a thermal exchange length

$$
l_{\mathrm{thex}}=\sqrt{\frac{A}{J_{s} H_{t h}}}
$$

where $H_{\mathrm{th}}$ is the strength of the thermal field

$$
H_{\text {th }}=\sqrt{\frac{2 \alpha k_{B} T}{\Delta t \gamma J_{s} l^{3}}} .
$$

Here $\Delta t$ is the time step of the time integration method. The definition of the thermal exchange length is well justified by the fact that the characteristic lengths are always sqrt(A/energy density), whereas the exchange, e.g. A, wants to align the magnetic moments parallel. The quantity in the denominator is associated with an effect that causes a nonuniform magnetization, for example anisotropy in a domain wall, the denominator is the energy density of this effect. So, in our case the denominator is, $J_{s}^{*} H_{t h}$.

Fig. 1 gives the thermal exchange length of different temperatures as a function of the spatial correlation length, $l$, which equals the cell size since the thermal noise is added for each cell. In analogy to the results of Rave and coworkes [5], we can guess that the numerical results will be independent of the cell size if the cell size, $l$, is smaller than $l_{\text {thex. }}$ In order to model white noise as good as possible, we would like to choose the spatial correlation length as small as possible on a given grid. Thus we arrive at $l=l_{\text {thex }}$, given by the solid line in Fig. 1. It becomes immediately clear that a computational grid fulfills the condition $l<l_{\text {thex }}$ only within a small range of the parameters. If the temperature increases the thermal field becomes larger. 
The thermal field then can override the exchange field. As a consequence the magnetization becomes inhomogeneous at a very small length scale and a fine mesh is required to resolve these small scale fluctuations of the magnetization.

Fig. 2 gives the average magnetization of a cubic Co particle Co $\left(J_{\mathrm{s}}=1.76 \mathrm{~T}, A=1.3 \times 10^{-11}\right.$ $\mathrm{J} / \mathrm{m}, K_{\mathrm{u}}=4.5 \times 10^{5} \mathrm{~J} / \mathrm{m}^{3}$ ) as a function of the cell size for different temperatures. The magnetization parallel to the anisotropy direction is averaged over a time period of $50 \mathrm{~ns}$. The result becomes independent of the mesh size for low temperature where the cell size is smaller than the thermal exchange length. A similar result holds for the relaxation time of the magnetization. To lower the energy barrier an external field of $60 \%$ of the anisotropy field is applied. The edge length of the cubic particle was $8 \mathrm{~nm}$. Fig. 3 compares the calculated switching times as a function of the mesh size for different temperatures. Again only for low temperatures the cell size becomes smaller than the thermal exchange length thus independent of the mesh size.

\section{Summary}

Thermal noise causes spatial fluctuations of the magnetization. Below the thermal exchange length the magnetization remains uniform owing to ferromagnetic exchange interactions. In order to take into account the non-uniform distribution of the magnetization caused by thermal fluctuations, the computational cells have to be smaller than the thermal exchange length. The thermal exchange length decreases with increasing temperature but also depends on the Gilbert damping constant, the time step, and the cell size. 


\section{Acknowledgements}

This work was supported by the Austrian Science Fund (Y-132PHY).

\section{References}

[1] Y. Nakatani, Y. Uesaka, N. Hayashi, and H. Fukushima, J. Magn. Magn. Mater. 168, (1997) 347.

[2] J. L. Garcia-Palacios, F. J. Lazaro, Phys. Rev. B 58 (1998) 14937.

[3] K. Zhang, D. R. Fredkin, J. Appl. Phys. 85 (1999) 5208.

[4] N.G. van Kampen, Stochastic processes in physics and chemistry (Norh-Holland, Amsterdam, 1992).

[5] W. Rave, K. Ramstock, and A. Hubert, J. Magn. Magn. Mater. 183 (1998) 329. 


\section{Figure Captions}

Figure 1: Thermal exchange length, $l_{\text {thex }}$, as a function of the spatial correlation length, $l$, for different temperatures and a Gilbert damping constant $\alpha=0.1$ and a time step $\Delta t=0.01 \mathrm{ps}$. The computational grid is fine enough to resolve the fluctuations in the magnetization if the mesh size $h=l$ (solid line) is smaller the $l_{\text {thex }}$.

Figure 2: Average magnetic polarization of a cubic Co particle with an edge length of $8 \mathrm{~nm}$ at different temperatures as a function of the cell size (Gilbert damping constant $\alpha=0.1$ and time step $\Delta t=0.01 \mathrm{ps}$ ). The arrows indicate the critical length at which the mesh size exceeds the thermal exchange length.

Figure 3: Relaxation time of a cubic Co particle with an edge length of $8 \mathrm{~nm}$ at an applied field of $60 \%$ of the anisotropy field as a function of the cell size (Gilbert damping constant $\alpha$ $=0.1$ and time step $\Delta t=0.01 \mathrm{ps})$. The arrows indicate the critical length at which the mesh size exceeds the thermal exchange length. 


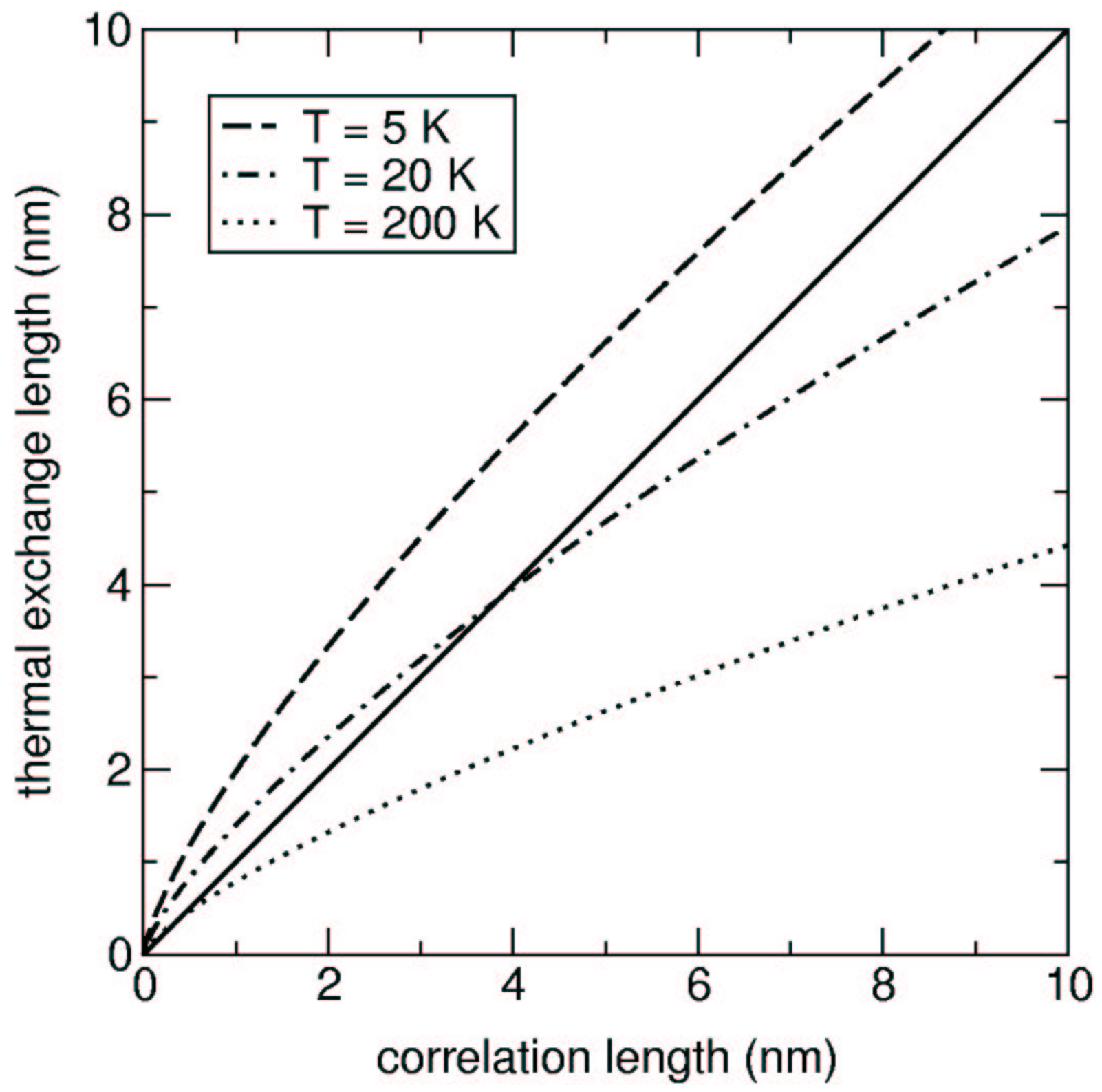

Fig. 1 


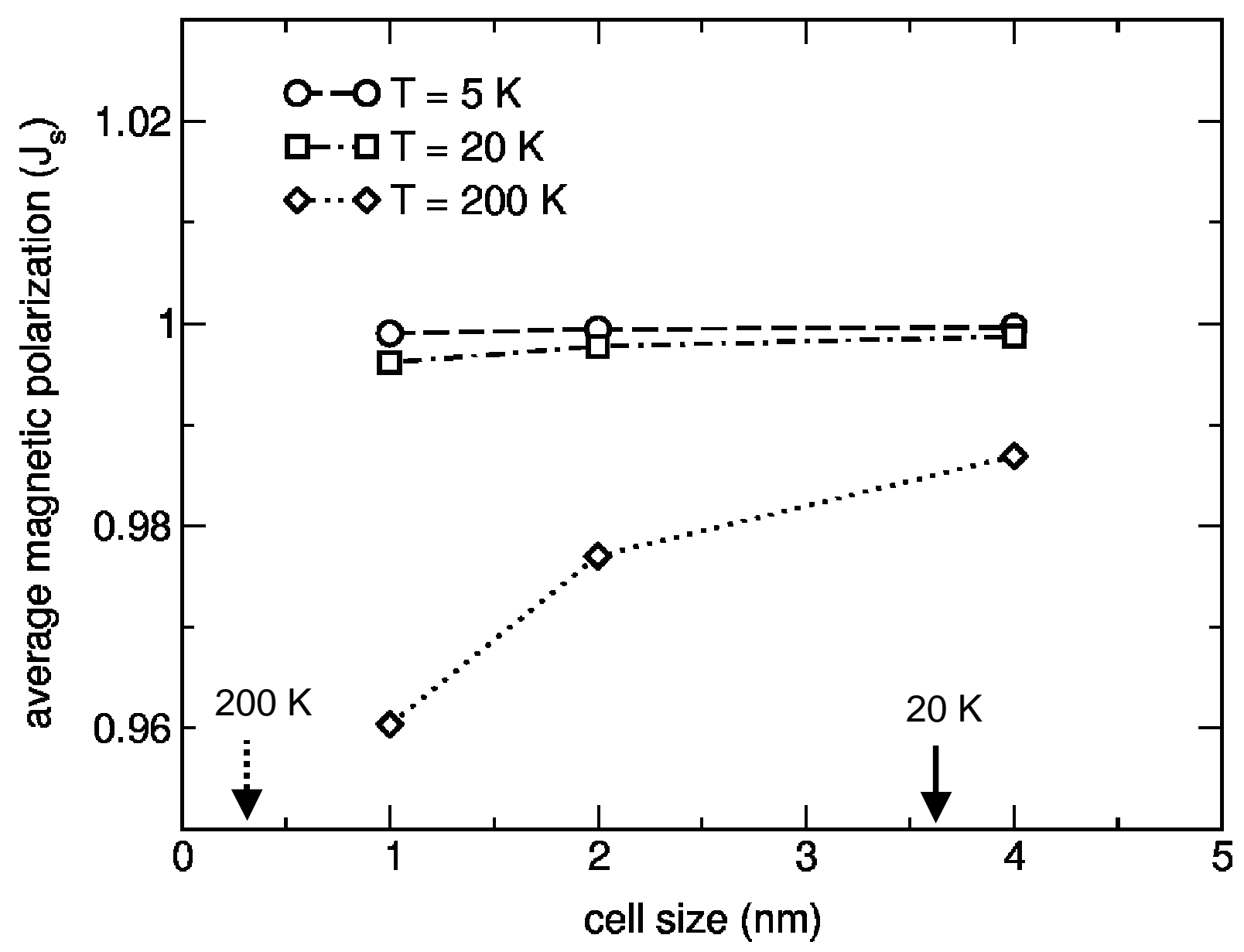

Fig. 2 


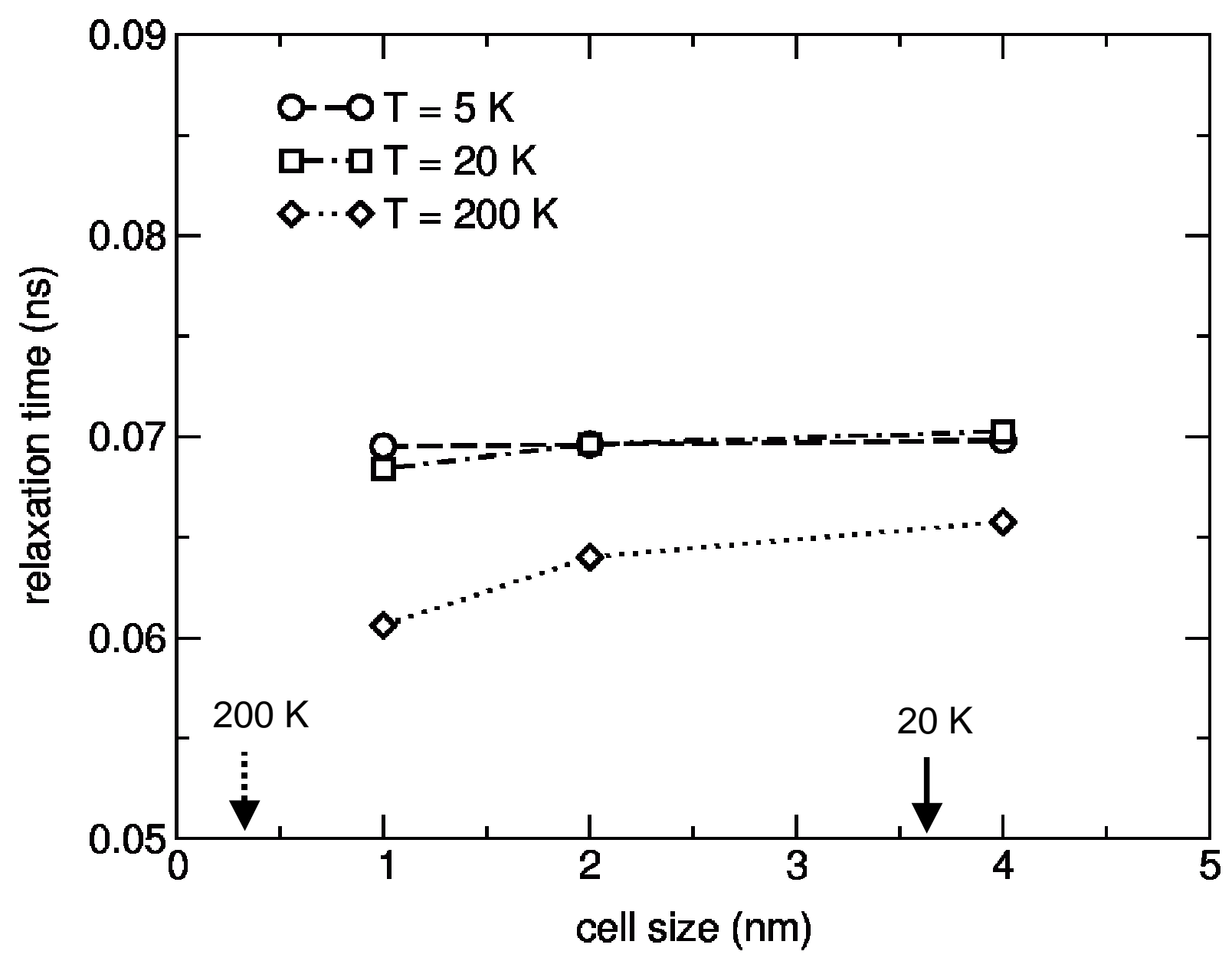

Fig. 3 